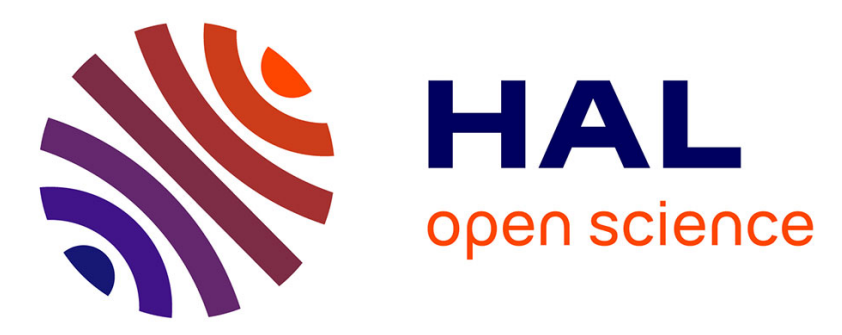

\title{
A fast identification algorithm for systems with delayed inputs
}

\author{
Kaouther Ibn Taarit, Lotfi Belkoura, Mekki Ksouri, Jean-Pierre Richard
}

\section{To cite this version:}

Kaouther Ibn Taarit, Lotfi Belkoura, Mekki Ksouri, Jean-Pierre Richard. A fast identification algorithm for systems with delayed inputs. International Journal of Systems Science, 2011, 42 (3), pp.449-456. inria-00437377

\section{HAL Id: inria-00437377 \\ https://hal.inria.fr/inria-00437377}

Submitted on 30 Nov 2009

HAL is a multi-disciplinary open access archive for the deposit and dissemination of scientific research documents, whether they are published or not. The documents may come from teaching and research institutions in France or abroad, or from public or private research centers.
L'archive ouverte pluridisciplinaire HAL, est destinée au dépôt et à la diffusion de documents scientifiques de niveau recherche, publiés ou non, émanant des établissements d'enseignement et de recherche français ou étrangers, des laboratoires publics ou privés. 


\title{
A fast identification algorithm for systems with delayed inputs
}

\author{
Kaouther Ibn Taarit ${ }^{a, b, d, e, f *}$, Lotfi Belkoura ${ }^{a, c, f, d}$, Mekki Ksouri ${ }^{e}$ and Jean-Pierre Richard ${ }^{a, b, f, d}$ \\ ${ }^{a}$ Université Lille Nord de France; ${ }^{b}$ ECLille, 59655 Villeneuve d'Ascq, France; ${ }^{c}$ USTL, Université des \\ Sciences et Technologies de Lille, 59650 Villeneuve d'Ascq, France; ${ }^{d}$ Alien Project (INRIA Lille-Nord \\ Europe); ${ }^{e}$ Unité de recherche ACS, ENIT, 1002 Tunis, Tunisia; ${ }^{f}$ Laboratoire LAGIS (CNRS, UMR \\ $8146)$
}

(v3.2 released March 2008)

\begin{abstract}
A fast identification algorithm is proposed for systems with delayed inputs. It is based on a non-asymptotic distributional estimation technique initiated in the framework of systems without delay. Such technique leads to simple realization schemes, involving integrators, multipliers and piecewise polynomial or exponential time functions. Thus, it allows for a real time implementation. In order to introduce a generalization to systems with input delay, three simple examples are presented here. The first illustration is a first order model with delayed input and noise. Then, a second order system driven through a transmission line is considered. A third example shows a possible link between simultaneous identification and generalized eigenvalue problems.
\end{abstract}

Keywords: Input delay systems; Identification; Generalized eigenvalue problem

\section{Introduction}

Time delay is a classical source of complexity in control problems. Actuators, sensors, computers, field networks and wireless communications that are involved in feedback loops unavoidably introduce delays, which are generally time-varying. Despite numerous advances in this field, delay remains a theoretical and practical challenge (see, e.g., the survey Richard (2003)), for systems controlled over networks in particular. Among the numerous open problems, the real-time delay identification is most crucial. On the one hand, various powerful control techniques (predictors, flatness-based predictive control, finite spectrum assignments, observers, ...) may be applied, provided the delay is known. On the other hand, the existing identification techniques for timedelay systems (see, e.g., Ren et al. (2005) for a modified least squares technique, and a survey in (Belkoura et al. 2004)) generally suffer from poor speed performance. This paper is a synthesis of the distributional approach for input delay and parameter estimation that was recently proposed in (Belkoura et al. 2006, 2008, 2009). Let us recall that those techniques are not asymptotic (in other words, they allow for a finite-time convergence) and do not need any statistical knowledge of the noises corrupting the data (see, e.g., Fliess et al. (2004) for applications to nonlinear state estimation, Fliess et al. (2005) for linear and nonlinear diagnosis). Several successful laboratory experiments have already been performed; see, e.g., (Beltrán-Carvajal et al. (2005)), (Pereira et al. (2009)), (Villagra et al. (2009)) and (Gédouin et al. (2009)).

We adopt in this paper a distributional formulation (compare with Fliess et al. (2003)) from which the parameters as well as the input delays may be easily estimated. The identification procedure employs elementary input signals, i.e., piecewise constant or more general polynomial time functions.

\footnotetext{
* Corresponding author . Email: Kaouther.IbnTaarit@enit.rnu.tn (C) 200x Taylor \& Francis DOI: $10.1080 / 0020717 \mathrm{YYxxxxxxxx}$

http://www.informaworld.com
} 
This paper is organized as follows. Section 2 gives the main theoretical tools we shall need for our identification problem. Section 3 and 4 focuse respectively on identification problem of a first order system with noisy delayed input and on a local identification with application to systems with transmission delays. Section 5 aims at showing how simultaneous parameters and delays identification can be formulated in terms of generalized eigenvalue problem.

\section{Mathematical framework}

\section{$2.1 \quad$ Notations}

We recall here some standard definitions and results from distribution theory Schwartz (1966) and fix the notations we shall use in the sequel. Let $\Omega$ be an open subset of $\mathbb{R}$. The space of $C^{\infty}$ functions having compact support in $\Omega$ is denoted by $\mathcal{D}(\Omega), \mathcal{D}^{\prime}(\Omega)$ is the space of distributions on $\Omega$, i.e., the space of continuous linear functionals on $\mathcal{D}(\Omega)$. The complement of the largest open subset of $\Omega$ in which a distribution $T$ vanishes is called the support of $T$ and is written $\operatorname{supp} T$. Write $\mathcal{D}_{+}^{\prime}$ (resp. $\mathcal{E}^{\prime}$ ) the space of distributions with support contained in $[0, \infty)$ (resp. compact support). It is an algebra with respect to convolution with identity $\delta$, the Dirac distribution. When concentrated at a point $\{\tau\}$, the latter distribution $\delta(t-\tau)$ is written $\delta_{\tau}$. A distribution is said to be of order $r$ if it acts continuously on $C^{r}$-functions but not on $C^{r-1}$-functions. Measures and functions are of order 0 . Functions are considered through the distributions they define and are therefore indefinitely differentiable. If $y$ is a continuous function except at a point $a$ with a finite jump $\sigma_{a}$, its derivative $d y / d t$ is $d y / d t=\dot{y}+\sigma_{a} \delta_{a}$, where $\dot{y}$ is the distribution stemming from the usual derivative of $y$. Derivation, integration and translation can be formed from the convolution products $\dot{y}=\delta^{(1)} * y, \quad \int y=H * y, \quad y(t-\tau)=\delta_{\tau} * y$, where $\delta^{(1)}$ is the derivative of the Dirac distribution, and $H$ is the familiar Heaviside function. With a slight abuse of notations, we shall write $H^{k} y$ the $k^{t h}$-order iterated integration of $y$ and, more generally, $T^{k}$ the iterated convolution product of order $k$. For $S, T \in \mathcal{D}_{+}^{\prime}$, supp $S * T \subset \operatorname{supp} S+\operatorname{supp} T$, where the sum in the right hand side is defined by $\{x+y ; x \in \operatorname{supp} S, y \in \operatorname{supp} T\}$. Finally, with no danger of confusion, we shall sometimes denote $T(s), s \in \mathbb{C}$, the Laplace transform of $T$.

\subsection{Background}

Multiplication of two distributions (say $\alpha$ and $T$ ) is not always defined. This operation makes however sense if one of the two terms is a smooth function. Several properties can be derived from such products. In particular, the next Theorem is the key result from which most of the parameters (including the delays) can be estimated.

Theorem 2.1: If $T$ has a compact support $K$ and is of finite order $m, \alpha T=0$ whenever $\alpha$ and all its derivatives of order $\leq m$ vanish on $K$ (Schwartz 1966).

The following examples illustrate this statement when $\alpha$ is a polynomial or exponential function, and $T$ a singular distribution. Note that, in forming the product $\alpha T$, the delay $\tau$ involved in the argument $T(t-\tau)$ now appears also as a coefficient.

$$
\begin{gathered}
t \delta=0, \quad\left(1-e^{-\gamma t}\right) \delta=0, \\
(t-\tau) \delta_{\tau}=0, \quad\left(1-e^{-\gamma(t-\tau)}\right) \delta_{\tau}=0 .
\end{gathered}
$$

The usual Leibniz rule $(\alpha T)^{\prime}=\alpha^{\prime} T+\alpha T^{\prime}$ remains valid. We shall make use of another property involving both multiplication by $t^{n}$ or $e^{-\gamma t}$ and the convolution product, in case one of the two 
distributions ( $S$ or $T$ ) is of compact support:

$$
\begin{gathered}
t^{n}(S * T)=\sum_{k=0}^{n} C_{n}^{k}\left(t^{k} S\right) *\left(t^{n-k} T\right), \\
e^{-\gamma t}(S * T)=e^{-\gamma t} S * e^{-\gamma t} T .
\end{gathered}
$$

The $C_{n}^{k}$ are the familiar binomial coefficients. Combining Leibniz rule and (1) with $S=\delta^{(p)}$ and $T=y$ allows us to transform terms of the form $t^{n} y^{(p)}$ or $v=e^{-\gamma t} y^{(p)}$ into linear derivatives sums of products $t^{k} y$. Setting $z_{i}=t^{i} y$ and $v=e^{-\gamma t} y$ yields

$$
\begin{gathered}
t^{3} y^{(2)}=t^{3}\left(\delta^{(2)} * y\right)=-6 z_{1}+6 z_{2}^{(1)}-z_{3}^{(2)}, \\
e^{-\gamma t} y^{(2)}=\gamma^{2} v+2 \gamma v^{(1)}+v^{(2)}
\end{gathered}
$$

where, $z_{1}=t y, z_{2}^{(1)}$ is the first derivative of $z_{2}=t^{2} y$, and $z_{3}^{(2)}$ is the second derivative of $z_{3}=t^{3} y$.

Note that integrating twice these expressions results in integration by parts formula.

\section{Single delay and parameter estimation}

\subsection{Identification method}

Consider a first order system with a delayed input ${ }^{1}$ :

$$
\dot{y}+a y=y(0) \delta+\gamma_{0} H+b u(t-\tau),
$$

where $\gamma_{0}$ is a constant perturbation, $a, b$, and $\tau$ are constant parameters. The coefficient $a$ is assumed to be known for the moment. Consider also a step input $u=u_{0} H$. A first order derivation yields

$$
\ddot{y}+a \dot{y}=\varphi_{0}+\gamma_{0} \delta+b u_{0} \delta_{\tau},
$$

where $\varphi_{0}=(\dot{y}(0)+a y(0)) \delta+y(0) \delta^{(1)}$, of order 1 and support $\{0\}$, contains the contributions of the initial conditions. By virtue of Theorem 2.1, multiplication of equation (6) by a function $\alpha$ such that $\alpha(0)=\alpha^{\prime}(0)=0, \alpha(\tau)=0$ cancels the right side. The choice of the polynomial function $\alpha(t)=t^{3}-\tau t^{2}$ results in:

$$
\begin{aligned}
t^{3}[\ddot{y}+a \dot{y}] & =\tau t^{2}[\ddot{y}+a \dot{y}], \\
b u_{0} t^{3} \delta_{\tau} & =b u_{0} \tau t^{2} \delta_{\tau} .
\end{aligned}
$$

The delay $\tau$ becomes available after $k \geq 2$ successive integrations. More precisely, since supp $H^{k} \delta_{\tau} \subset(\tau, \infty)$, equation (8) shows that all the obtained functions will vanish on $(0, \tau)$ and the delay is consequently not identifiable on this interval. Conversely, those functions being nonzero for all $t>\tau$, the delay is everywhere identifiable on $(\tau, \infty)$. We therefore obtain from (7):

$$
\tau=\frac{H^{k}\left(w_{0}+a w_{3}\right)}{H^{k}\left(w_{1}+a w_{2}\right)}, \quad t>\tau
$$

${ }^{1}$ Such systems, where the delays only appear in the control variables, are most common in practice. See Fliess et al. (2002) for their theoretical background, and their control. 


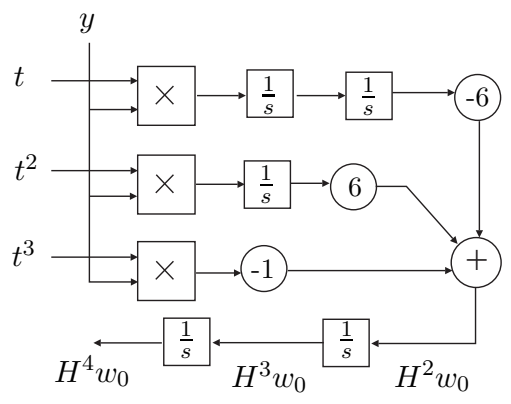

Figure 1. Realization scheme of $H^{k} w_{0}$.

where, by virtue of equation (1) and recalling the notation $z_{i}=t^{i} y$ of the previous Section, we set:

$$
\begin{aligned}
& w_{0}=t^{3} y^{(2)}=-6 z_{1}+6 z_{2}^{(1)}-z_{3}^{(2)}, \\
& w_{1}=t^{2} y^{(2)}=-2 z_{0}+4 z_{1}^{(1)}-z_{2}^{(2)} \\
& w_{2}=t^{2} y^{(1)}=2 z_{1}-z_{2}^{(1)} \\
& w_{3}=t^{3} y^{(1)}=3 z_{2}-z_{3}^{(1)} .
\end{aligned}
$$

These coefficients show that $k \geq 2$ integrations avoid any derivation in the delay identification. Due to the non identifiability on $(0, \tau)$, the delay $\tau$ is set to zero until the numerator or denominator in the right hand side of (9) reaches a significant nonzero value. The algorithm given in (9) only requires the values of $a$ and of the output $y$. If $a$ is also unknown, the same approach may be utilized for a simultaneous identification. The following relation is easily derived from (7):

$$
\tau\left(H^{k} w_{1}\right)+a \tau\left(H^{k} w_{2}\right)-a\left(H^{k} w_{3}\right)=H^{k} w_{0}
$$

A linear system with unknown parameters $(\tau, a \tau, a)$ is obtained by using different integration orders

$$
\left(\begin{array}{ccc}
H^{2} w_{1} & H^{2} w_{2} & H^{2} w_{3} \\
H^{3} w_{1} & H^{3} w_{2} & H^{3} w_{3} \\
H^{4} w_{1} & H^{4} w_{2} & H^{4} w_{3}
\end{array}\right)\left(\begin{array}{c}
\hat{\tau} \\
\hat{a \tau} \\
-\hat{a}
\end{array}\right)=\left(\begin{array}{c}
H^{2} w_{0} \\
H^{3} w_{0} \\
H^{4} w_{0}
\end{array}\right) .
$$

The parameters $(\hat{\tau}, \hat{a} \hat{\tau},-\hat{a})$ to be estimated are obtained from the resolution of this last linear system (11). Figure 1 shows a partial realization scheme corresponding to the term $H^{k} w_{0}$. Each of the terms, $H^{k} w_{i}(i=0, \cdots, 3)$, involved in (9) and (11) are realized by using the same principle.

The resulting numerical simulations are shown in Figure 2 for the parameters $y(0)=0.3, a=$ $2, \tau=0.6, \gamma_{0}=2, b=1, u_{0}=1$. For the previous identifiability reason, the obtained linear system may be not consistent for $t<\tau$. Moreover, and unlike the single delay case, a local loss of identifiability (see Belkoura (2005)) may occur for $t>\tau$ as suggested in Figure 2 for $t \approx 1.7 \mathrm{~s}$. Multiplying $(6)$ by other smooth function permits the estimation of other parameter combinations. By setting, for instance, $\alpha(t)=t(t-\tau)$ and by using the same previous techniques we obtain:

$$
H^{k} t(\ddot{y}+a \dot{y}) \tau-H^{k} \tau y(0)=H^{k} t^{2}(\ddot{y}+a \dot{y})
$$

from which both delay and initial condition may be identified. The only coefficient for which 


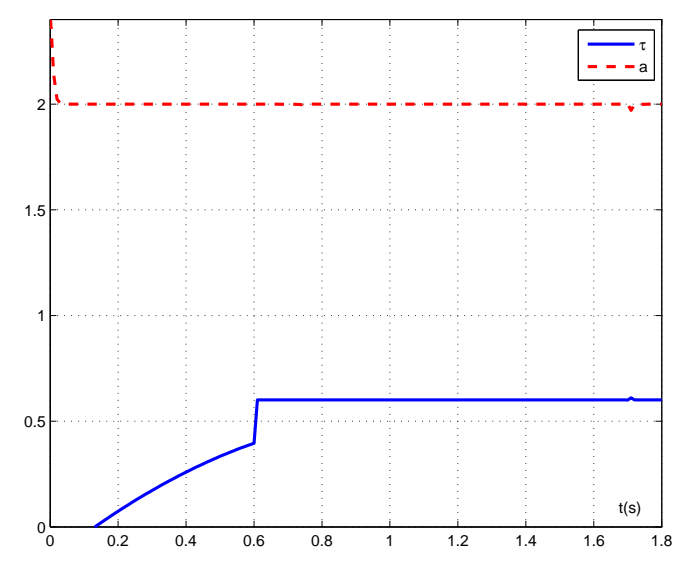

Figure 2. Simultaneous identification from (10).
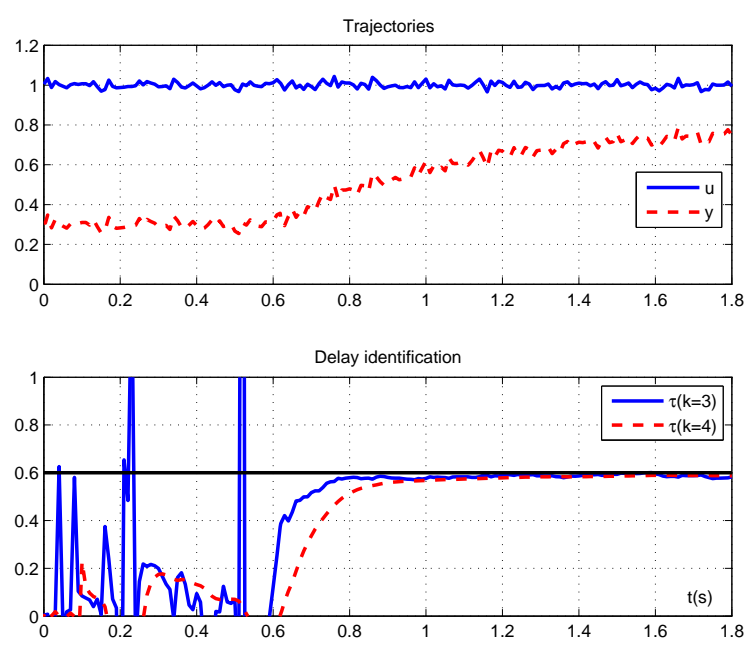

Figure 3. Delay identification with noise in the data.

the explicit value of $\tau$ is required is its associated parameter $b$. Finally, due to the fast convergence of the algorithms, we may also consider a separate procedure in which the undelayed terms are firstly identified and used afterward for the delay identification.

\subsection{Robustness with regard to noise}

As mentioned in Section 2.2, iterated convolutions using $H^{k}$ result in nothing but integration by part formulae. With noisy data, integration with $H(s)=1 / s$ may be replaced by any strictly proper transfer function and particularly by low pass filters such as $T(s)=1 /(\gamma s+1)$. The following simulations show favorable robustness properties of the proposed delay identifier with respect to noise corrupted data. The input and output white noise perturbations powers were taken to be of amplitude $2 \times 10^{-6}$ and $4 \times 10^{-6}$ respectively, while $\gamma=2$ was used for the filter.

Remark 1: See Fliess (2006) for a nonstandard analysis of noises which explains the above robustness results. Another possible explanation of the convergence may be deduced via the power spectrum of the additional terms that appear in (9) when the output $y$ is replaced by a measurement $m=y+\nu$. The contribution of the noise $\nu$ is made through terms of the form 


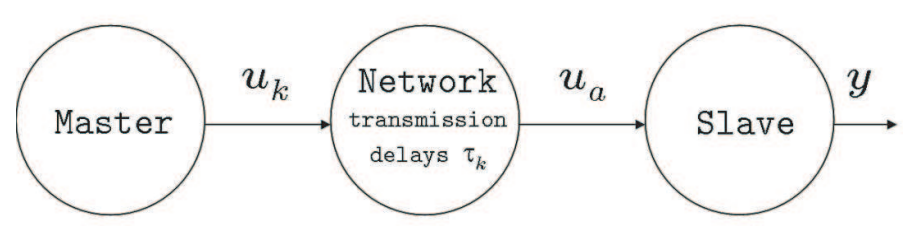

Figure 4. General structure of systems with transmission delay.

$T^{k}\left(\left(t^{p} \nu\right)^{(q)}\right)$ for which the power spectrum is given by

$$
|T(j \omega)|^{k}(j \omega)^{q} \frac{d^{p} \phi_{\nu \nu}(\omega)}{d \omega^{p}} .
$$

If the noise spectrum $\phi_{\nu \nu}(\omega)$ is assumed to be constant on a sufficiently large strip $-L<\omega<L$ (which is the case for white noise approximations), and by virtue of the strict causality obtained with $k>q$, these quantities, and hence the noise effects remain negligible.

\section{Local identification with application to systems with transmission delay}

The input delay of the previous Section has been estimated because it also corresponded to the commutation instant of the right hand side $\gamma_{0} H+b u_{0} H(t-\tau)$ of (5). Based on this observation, our techniques may be extended to a possible infinite number $K$ of delays by means of one of the three following approaches:

(1) A multiplication with the $C^{\infty}$-function $t^{2}\left(t-h_{1}\right) \cdots\left(t-h_{K}\right)$, if $K$ is finite,

(2) a recursive identification,

(3) a local identification if one assumes a lower bound for two successive delays, i.e., $h_{k+1}-h_{k}>$ $\Delta$. In such case $K$ may also be infinite.

The first case may however lead to a large size linear system for which the delays remain unknown until $t>h_{K}$, while error propagations may result from the second case. We next consider the third case described by Figure 4 and assume that a discrete reference signal $\left\{u_{k}\right\}$ with fixed period $T$ is sent from a Master to a continuous process (Slave) for which each data is hold until the next step. Due to the delay $\tau_{k}$ introduced by the transmission line, the actual input of the process will consist in a piecewise constant signal of the form

$$
u_{a}=\sum_{k=0}^{\infty} u_{k} \chi_{k}
$$

where $\chi_{k}$ denotes the characteristic function of the interval $\left[k T+\tau_{k},(k+1) T+\tau_{k+1}\right]$. Based on the output observations, we propose an on-line identification scheme of the time-instants $k T+\tau_{k}$. By this way, if the period $T$ is known and if Master and Slave share the same time origin, then the transmission delays $\tau_{k}$ become available on-line from the only measurements of $y$. The estimation of these transmission delays is important for Networked Control Systems. When the sent packets include time-stamps, the receiver may estimate the delay's duration and take appropriate corrective actions (see, e.g., Hespanha et al. (2007), Zampieri (2008), Yang et al. (2008) and Zhang et al. (2008)). When such time-stamps are not available (for instance, in some Bluetooth devices, the packets content is not modifiable by the user), our method is still applicable. For simplicity reasons, the process under consideration consists in a second order linear system

$$
\ddot{y}+a_{1} \dot{y}+a_{0} y=\varphi_{0}+\gamma_{0} H+b u_{a}
$$



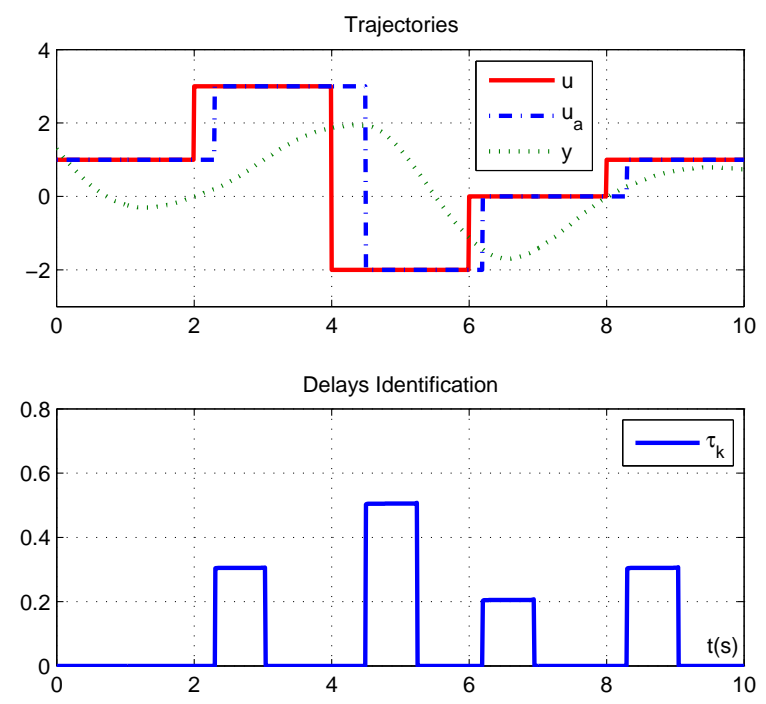

Figure 5. Trajectories and delays identification.

where $\gamma_{0} H$ is a constant perturbation, and $\varphi_{0}$ (of order 1 and support $\{0\}$ ) contains the initial conditions. Following the previous section, derivation and multiplication by $t^{3}(t-\lambda)$ yields

$$
t^{3}(t-\lambda)\left(y^{(3)}+a_{1} y^{(2)}+a_{0} y^{(1)}\right)=b \sum_{k=0}^{\infty} \sigma_{k}\left(h_{k}^{4}-\lambda h_{k}^{3}\right) \delta_{h_{k}},
$$

where we have denoted $h_{k}=k T+\tau_{k}$ and $\sigma_{k}$ the jumps of $u_{a}$ at $h_{k}$. Using the assumption $h_{k+1}-h_{k}>\Delta$, a "local" integration is now considered with $T(s)=\left(1-e^{-\Delta s / 3}\right) / s$ instead of $H(s)=1 / s$. Here, suppT $\subset(0, \Delta / 3)$ and the coefficient 3 also represents the number of integrations required in order to ensure causality of the identification scheme. This results in

$$
T^{3} t^{3}(t-\lambda)\left(y^{(3)}+a_{1} y^{(2)}+a_{0} y^{(1)}\right) \triangleq N-\lambda D,
$$

where, from the right hand side of (16), and by virtue of the support of a convolution product given in Section 2.1, one has

$$
\begin{aligned}
& \operatorname{supp} T^{3} \delta_{h_{k}} \subset\left(h_{k}, h_{k}+\Delta\right), \\
& \operatorname{supp}(N, D) \subset\left\{\left(h_{k}, h_{k}+\Delta\right) \mid k=1,2, \cdots\right\} .
\end{aligned}
$$

On each of the latter intervals, the commutation instant $h_{k}$, and hence the delay $\tau_{k}$ are therefore obtained from the relation

$$
\lambda=h_{k}=k T+\tau_{k}=N / D
$$

As in the previous section, terms of the form $T^{k} t^{l} y^{(q)}$ involved in the expressions of $N$ and $D$ are implemented by considering first the development of $t^{l} y^{(q)}$ described in (1) and (3) and then the integration with $T^{k}$. A simulation result is given in Figure 5 with the parameters $a_{0}=2$, $a_{1}=1, b=1, y(0)=1.3, \dot{y}(0)=-2.3, \Delta=2$ and the delays $\left\{\tau_{k}\right\}=\{0.3,0.5,0.2,0.3\}$.

Clearly, the quality of the estimation depends on the choice of the support $\Delta$. Our numerical investigations, using various choices of the $\Delta$ value, pointed out that a large support $\Delta$ improves the estimation and shows more interest in the noisy case. However, due to the constraint $\Delta<\min \left(h_{k+1}-h_{k}\right)$, increasing $\Delta$ cannot be done without some additional information on the 
expected times $h_{k}$. Note that the same study could be conducted with unknown parameters $a_{0}$ and $a_{1}$ as well.

\section{Simultaneous delay and parameters identification as a generalized eigenvalue problem}

\subsection{Problem statement}

Unlike the undelayed case where the parameters are identified from a linear system of equations, this last example, taken from the aeronautic field (Veysset et al. 2006) and improved, shows how combined delay-and-parameter identification can be formulated in terms of a generalized eigenvalue problem. The example consists in a system with both delayed and undelayed inputs and in which each of the corresponding effects results in a first order transfer:

$$
y=\left[\frac{k_{w 0}+k_{w 1} s}{1+\tau_{w} s}+\frac{k_{t 0}+k_{t 1} s}{1+\tau_{t} s} e^{-\tau s}\right] u
$$

Some simple computations show that the right-hand side of the resulting differential equation can be canceled by means of a multiplication with a function $\alpha$ such that $\alpha^{(k)}(0)=\alpha^{(k)}(\tau)=0$ for $k=0,1,2$. The choice of the exponential function

$$
\alpha(t)=\left(1-e^{-\gamma t}\right)^{3}\left(1-e^{-\gamma(t-\tau)}\right)^{3}
$$

results in

$$
(1-x)^{3}(1-\lambda x)^{3}\left(a_{2} y^{(3)}+a_{1} y^{(2)}+y^{(1)}\right)=0,
$$

where for ease of notations we denoted $x=e^{-\gamma t}, \lambda=e^{-\gamma \tau}$, and $a_{2}=\tau_{t} \tau_{w}, a_{1}=\tau_{t}+\tau_{w}$. Note that the latter equation non longer requires the knowledge of the gains $k_{w 0}, k_{w 1}, k_{t 0}$ and $k_{t 1}$. Here again, $k \geq 3$ successive integrations (or a convolution with $H^{k}$ ) result in functions equality from which the delay $\tau$ and the coefficients $\tau_{w}, \tau_{t}$ become available. More precisely, equation (23) combined with integrations leads to the following formulation:

$$
\left(A_{0}+\lambda A_{1}+\lambda^{2} A_{2}+\lambda^{3} A_{3}\right)\left(\begin{array}{c}
a_{2} \\
a_{1} \\
1
\end{array}\right)=0
$$

where the matrices entries are obtained by means of integration by part formula and are given by:

$$
\begin{aligned}
& A_{0}(i, j)=+1 H^{i+3}(1-x)^{3} y^{(4-j)} \\
& A_{1}(i, j)=-3 H^{i+3} x(1-x)^{3} y^{(4-j)} \\
& A_{2}(i, j)=+3 H^{i+3} x^{2}(1-x)^{3} y^{(4-j)} \\
& A_{3}(i, j)=-1 H^{i+3} x^{3}(1-x)^{3} y^{(4-j)}
\end{aligned}
$$

The identification problem is therefore transformed into a generalized eigenvalue problem (24) for which the delay is deduced from one of the eigenvalues (i.e. $\tau=-\log (\lambda) / \gamma$ ), while the coefficients $a_{2}$ and $a_{1}$ are obtained from the corresponding normalized eigenvector. Figure 6 shows the generalized eigenvalues of (24) (and more precisely $-\log (\lambda) / \gamma$ ) solved using the polyeig Matlab function and the parameters $\tau_{w}=0.6, k_{w 0}=2, k_{w 1}=0.5, \tau_{t}=0.4, k_{t 0}=0.7, k_{t 1}=0.1$, $\tau=0.5, \gamma=0.2$. The result clearly shows one constant eigenvalue corresponding to the unknown delay $\tau$. This value appears after a transitory phase for which the delay is not identifiable. We 


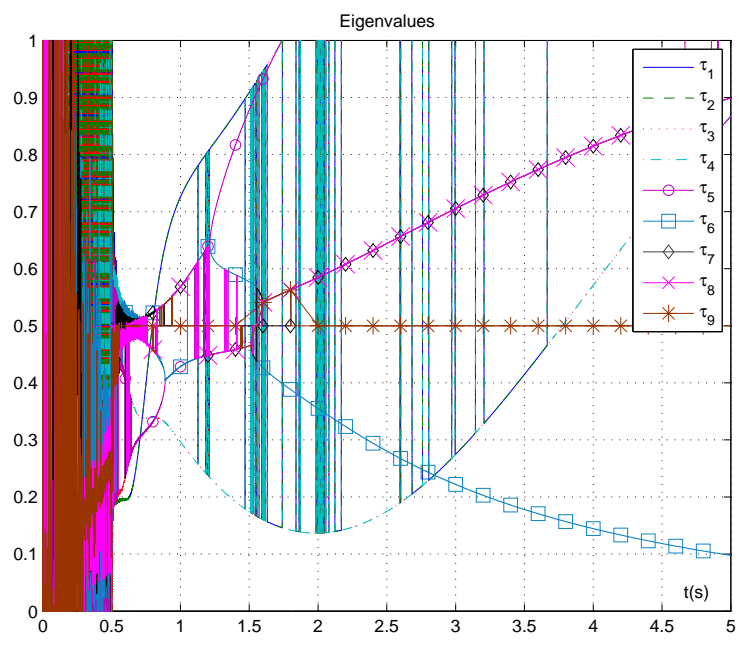

Figure 6. Eigenvalues of problem (24).

therefore get nine possible eigenvalues (resp. eigenvectors) of which one corresponds to the time delay (resp. coefficients $a_{2}$ and $a_{1}$ ) and the others are to be rejected. It is therefore necessary to design an on-line identification algorithm which allows to select the desired eigenpair. Such a procedure is a direct continuation of Belkoura et al. (2008) and is developed in the following section.

\subsection{Resolution of the spectral problem}

The previous section shows that the desired eigenpair satisfies the equation (24) for any $k \geq 3$. Therefore, finding this eigenpair is equivalent to select the eigenpair $(\hat{\lambda}, \underline{\hat{v}})$ that minimizes $\left\|\left(A_{0}+\hat{\lambda} A_{1}+\hat{\lambda}^{2} A_{2}+\hat{\lambda}^{3} A_{3}\right) \underline{\hat{v}}\right\|_{2}$, where $A_{i=0, \ldots 3}$ are typically rectangular $(m \times n)$ matrices. Clearly, more measurements (and thus more rows) imply better possible estimation. In order to understand the physical aspect of this eigenvalues, it is interesting to make the analytic computation of the eigenvalues of the first order system described by equation (5) under zero initial condition and without constant perturbation for the simple case of candidate function $\alpha(t)=(t-\tau)$. The results of the eigenvalues computation for the square pencil $(A, B)$ formed by lines $(1,2)$ and lines $(2,3)$ are respectively given by the systems of equations $(S 1)$ and $(S 2)$.

$$
(S 1)=\left\{\begin{array}{l}
\lambda_{1}=\tau \\
\lambda_{2}=\frac{t \tau+2 t a_{0}-4 a_{0} \tau-6 a_{0}^{2}-\tau^{2}}{\Delta_{1}}+\frac{\left(t^{2}+4 t a_{0}-t \tau+6 a_{0}^{2}-2 a_{0} \tau\right) \exp \left(-\frac{t-\tau}{a_{0}}\right)}{\Delta_{1}}
\end{array}\right.
$$

where, $a_{0}$ is the time constant and $\Delta_{1}=\left(t+2 a_{0}-\tau\right) \exp \left(-\frac{t-\tau}{a_{0}}\right)+t-\tau-2 a_{0}$, and

$$
(S 2)=\left\{\begin{array}{l}
\lambda_{2}=\tau \\
\lambda_{3}=\frac{\left(2 t^{2} a_{0}+12 t a_{0}^{2}-2 t a_{0} \tau\right) \exp \left(-\frac{t-\tau}{a_{0}}\right)}{\Delta_{2}}+\frac{\left(24 a_{0}^{3}-6 a_{0}^{2} \tau\right) \exp \left(-\frac{t-\tau}{a_{0}}\right)-t \tau-2 a_{0} t^{2}}{\Delta_{2}} \\
+\frac{8 t a_{0} \tau+2 \tau^{2} t+12 t a_{0}^{2}-\tau^{3}-6 a_{0} \tau^{2}}{\Delta_{2}}+\frac{-6 a_{0} \tau^{2}-24 a_{0}^{3}-18 a_{0}^{2} \tau}{\Delta_{2}}
\end{array}\right.
$$

where, $\Delta_{2}=\left(2 t a_{0}-2 a_{0} \tau+6 a_{0}^{2}\right) \exp \left(-\frac{t-\tau}{a_{0}}\right)-t^{2}+4 t a_{0}+2 \tau t-4 a_{0} \tau-6 a_{0}^{2}-\tau^{2}$.

The analytic computation of eigenvalues of the square pencil clearly shows that there are two distinct eigenvalues. For both, the first one is constant and corresponds to the time delay $\tau$, while the other is unstationar. This example shows that the unstationar eigenvalues does not 

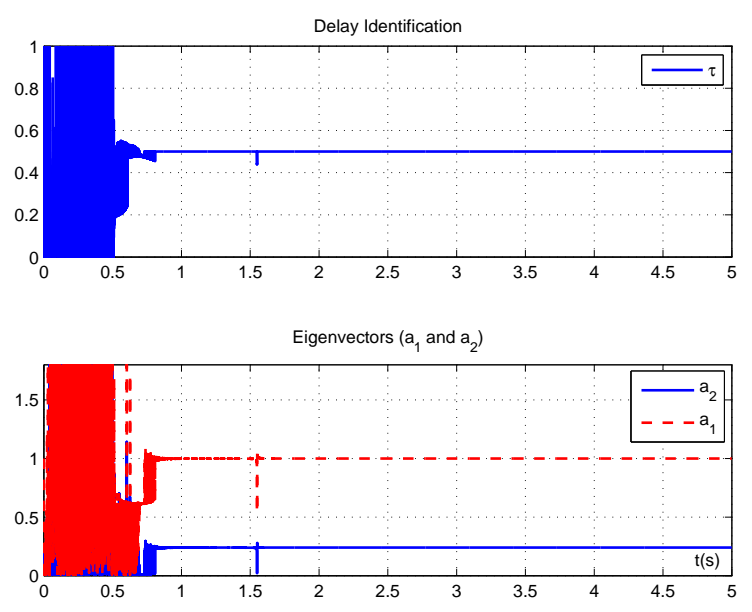

Figure 7. Estimated parameters $\tau, a_{1}$ and $a_{2}$.

satisfy the rectangular pencil.

The choice of the eigenvalue that fits the delay has been made by adding a line to square pencil that became rectangular. We do know as well that the delay satisfies this equation and so it cancels the whole rectangular pencil. That is why we've chosen the eigenvalue that minimizes $\|(A-\hat{\lambda} B) \underline{\hat{v}}\|_{2}$ where $A$ and $B$ are rectangular.

Figure 7 shows the simulation results for $k=5$ successive integrations. Due to non identifiability on $(0, \tau)$, parameters $\tau, a_{1}$ and $a_{2}$ have no significant values. Note also that a singularity occurred for $t \simeq 1.5 \mathrm{~s}$.

\subsection{Remark on the choice of the candidate function $\alpha(t)$}

The identification algorithms depend on the choice of the candidate function $\alpha(t)$. Indeed, this choice is linked to the Schwartz's theorem 2.1. Our first two examples involved polynomial functions, whereas the previous third example used exponential ones. More generally, any combination of the type $\alpha(t)=\alpha_{1}(t)-\lambda(\tau) \alpha_{2}(t)$, where, $\lambda(t)$ is a bijective function of $\tau$, can be proposed.

\section{Conclusion}

The original contribution of this paper is the simultaneous delay and parameters identification of a widespread class of systems. Simulated examples have been presented here, that show the efficiency of the approach. On these bases, we hope that the high speed convergence of our algorithms will now permit to treat simultaneously the on-line identification and control of time-delay systems in concrete applications. Our investigations pointed out that the candidate function $\alpha$ and the filter can be oriented with regard to the situation. Concerning the candidate function $\alpha(t)$, the polynomial functions are simple to manipulate but they raise robustness problems in the case of noisy data. That is why, in noisy situations, it is recommended to use the exponentional functions or a bounded function in general. Concerning the filter, as it was explained in Section 3.2, any low-pass filter $T(s)$ can be implemented and replace the basic integration $H(s)$, which increases the robustness with regard to noise. Identifiability issues, MIMO systems with partial state measurements, state delay estimation, as well as discrete-time extensions are under active investigation. 


\section{References}

Belkoura, L., Dambrine, M., Orlov, Y. and Richard, J.-P.(2004), "Identifiability and identification of linear systems with delays Advances in Time-Delay Systems," S.-I. Niculescu, K. Gu (Eds), Lect. Notes Comput. Sci. Engin., Springer, Berlin, vol. 38, pp. 123-135.

Belkoura, L. (2005), "Identifiability of systems described by convolution equations," Automatica, vol. 41, pp. 505-512.

Belkoura, L., Richard, J.-P. and Fliess, M. (2006), "On line identification of systems with delayed inputs," In MTNS'06, 16th Conference on Mathematical Theory of Networks and Systems, invited session Networked systems, Kyoto, Japan, July.

Belkoura, L., Richard, J.-P. and Fliess, M. (2008), "A convolution approach for delay systems Identification," 17th IFAC World Congress, Seoul, Korea, July 6-11.

Belkoura, L., Richard, J.-P. and Fliess, M. (2009), "Parameters estimation of systems with delayed and structured entries," Automatica, vol. 45, pp. 1117-1125.

Beltrán-Carvajal, F., Silva-Navarro, G., Sira-Ramírez, H., Quezada-Andrade, J.(2005), "Active vibration control using on-line algebraic identification of harmonic vibrations," Proc. Amer. Control Conf., Portland, OR.

Fliess, M., Marquez, R., Mounier, H.(2002), "An extension of predictive control, PID regulators and Smith predictors to some linear delay systems," Internat. J. Control, vol. 75, pp. 728743.

Fliess, M., Sira-Ramírez, H.(2003), "An algebraic framework for linear identification," ESAIM Contr. Optim. Cal. Variat., vol. 9, pp. 151-168.

Fliess, M., Sira-Ramírez, H.(2004), "Control via state estimations of some nonlinear systems," Proc. Symp. Nonlinear Control Systems (NOLCOS 2004), Stuttgart, (avalable: http://hal.inria.fr/inria-00001096).

Fliess, M., Join, C., Sira-Ramírez, H.(2005), "Closed-loop fault-tolerant control for uncertain nonlinear systems," Control and Observer Design for Nonlinear Finite and Infinite Dimensional Systems, T. Meurer, K. Graichen, E.D. Gilles (Eds), Lect. Notes Control Informat. Sci., Springer, Berlin, vol. 322, pp. 217-233.

Fliess, M.(2006), "Analyse non standard du bruit," C.R. Acad. Sci. Paris, ser. I, vol. 342, pp. 797-802.

Gédouin, P.-A., Join, C., Delaleau, E., Bourgeot, J.-M., Chirani, S.-A., and Calloch, S. (2009), "A new control strategy for shape memory alloys actuators," 8th European Symposium on Martensitic Transformations (ESOMAT 2009), Prague, Czech Republic, September 7-11.

Hespanha, J.P., Naghshtabrizi, P., Yonggang, Xu. (2007), "A Survey of Recent Results in Networked Control Systems," Proceedings of the IEEE, vol. 95, pp. 138-162.

Pereira, E., Trapero, J.-R., Daz, I.-M., Feliu, V.(2009), "Adaptive input shaping for manoeuvring flexible structures using an algebraic identification technique," Automatica, vol. 45, pp. 10461051.

Ren, X.M., Rad, A.B., Chan, P.T., Lo, W.L.(2005), "On-line identification of continuous-time systems with unknown time-delay," IEEE Trans. Automat. Contr., vol. 50, pp. 1418-1422.

Richard, J.-P. (2003), "Time-delay systems: an overview of some recent advances and open problems," Automatica, vol. 39, pp. 1667-1694.

Schwartz, L.(1966), "Théorie des distributions (2 $2^{\text {nd }}$ ed.)," Hermann, Paris.

Veysset, F., Belkoura, L., Coton, P., Richard, J.-P.(2006), "Delay system identification applied to the longitudinal flight of an aircraft through a vertical gust," Proc. $14^{\text {th }}$ Medit. Conf. Control Automat., Ancona, Italy.

Villagra, J., Dandrhéa-novel, B., Choi, S., Fliess, M., Mounier, H.(2009), "Robust stop-and-go control strategy: an algebraic approach for nonlinear estimation and control," International Journal of Vehicle Autonomous Systems, (avalable: http://hal.inria.fr/docs/00/41/94/45/PDF/IJVAS.pdf).

Yang,Y., Wang, Y., Shuang-Hua, Y.(2008), "Design of a networked control system with random 
transmission delay and uncertain process parameters," International Journal of Systems Science, vol. 39, pp. 1065-1074.

Zampieri, S.(2008), "Trends in Networked Control Systems," Proceedings of the 17th World Congress, the International Federation of Automatic Control, Seoul, Korea, July 6-11.

Zhang, Y., Chen, Y., Sheng, T.,Hesketh, T.(2008), "Fault detection and diagnosis of networked control system," International Journal of Systems Science, vol. 39, pp. 1017-1024.

\section{Biographies}

Kaouther Ibn Taarit was born in Tunis, Tunisia, in 1982. She received

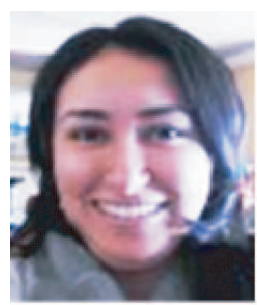
the engineering degree in Electrical Engineering and the M.S degree in Control and Signal Processing from the National School Engineering of Tunis in 2006 and 2007. Where, she is currently pursuing her Ph.D. degree in Department of Electrical Engineering at National School Engineering of Tunis (ENIT) and in Central school of Lille (ECLille). Her research interests include Time delay systems and Parametric identification problems.

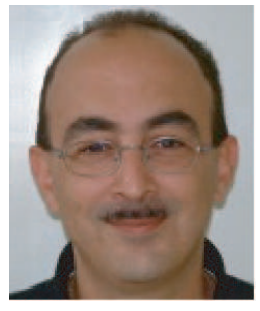

Lotfi Belkoura is currently an Assistant Professor at the University of Science and Technology of Lille, France. He received the M.S. Degree in 1989, the Ph.D. Degree in Automatic Control and the "Habilitation diriger des recherches" from the same University in 1993 and 2006. In 1999, he joined the Laboratory of Automatic control LAGIS, Computer Sciences and Signal of Lille, and in 2004 the Alien Project of the INRIA Institute. His main research interests include Time delay systems, Hybrid systems, Identification problems with applications to Transportation and Communication.

Mekki Ksouri received the M.A. degree in physics in the FST in Tunis

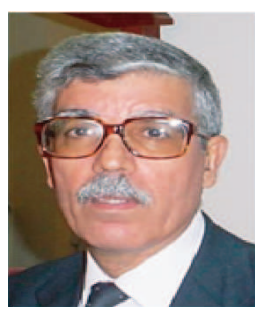
in 1973, the M.E. degree Ingenieur SupElec (Ecole Suprieure d'Electricite) in Paris 1973, the D.Sc. degree, and the Ph.D. degree from the University of Pierre and Marie Curie, Paris, France, in 1975 and 1977 respectively. He is Professor at the National School of Engineers of Tunis (ENIT). He was principal of the National Institute of Applied Sciences and Technology (INSAT) from 1999 to 2005, principal and founder of the High School of Statistics and Information Analysis from 2001 to 2005 and the High Institute of Technologic Studies from 1996 to 1999 and principal of The High Normal School of Technological Education from 1978 to 1990. He is also the author of 6 books. His activities focus on automatic control, robust control, and optimization in planning and scheduling, including implementation of fuzzy logic, neural nets, and genetic algorithms. He had received the Outstanding Contribution Award for Leadership in the Organization of the CIFA 2004 Symposium, Chevalier dans l'Ordre des Palmes Acadmiques France 2002, Outstanding Contribution Award IEEE SMC 1998. He is a senior member IEEE and he was the head and founder of many scientific associations (ATTNA, $\mathrm{ASET}, \ldots)$.

Jean-Pierre Richard was born in Montpellier (France) in 1956, Dipl. Eng.

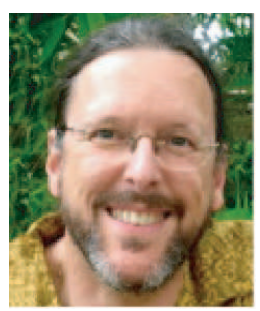
1979, M.Sc. in Electronics in 1979, Ph.D. in Automatic Control in 1981, D.Sc. in Physical Sciences in 1984 (University of Lille). He is presently a Professor at the Ecole Centrale de Lille (French "Grande Ecole") and the President of the research federation GRAISyHM (French acronym for Research Group in Integrated Automation and Man-Machine Systems, Nord-Pas-de-Calais Region, France). His research interests concern the control and estimation of complex processes and, more particularly, of time delay systems. Details and publications can be found on http://syner.ec-lille.fr/ richard/. 